\title{
The Size of a Photon
}

\author{
Richard A. Hutchin \\ Optical Physics Company, Simi Valley, USA \\ Email: rahutchin@opci.com
}

How to cite this paper: Hutchin, R.A. (2021) The Size of a Photon. Optics and Photonics Journal, 11, 121-131. https://doi.org/10.4236/opj.2021.115010

Received: March 22, 2021

Accepted: May 18, 2021

Published: May 21, 2021

Copyright $\odot 2021$ by author(s) and Scientific Research Publishing Inc. This work is licensed under the Creative Commons Attribution International License (CC BY 4.0).

http://creativecommons.org/licenses/by/4.0/

\begin{abstract}
This paper begins by exploring a useful and neglected detail of a photon-its physical size perpendicular to the direction of propagation in the same way as an atom or neutron has a physical size. Such a photon size would be quite separate from the cross-section of a photonic interaction, which depends on the material interacting. Such a perpendicular dimension of a photon will be invariant under Lorentz transform parallel to the light propagation direction and will thus be the same for all frequencies of light. This study also leads to new details about how a photon interacts, offering an explanation for the familiar physics where light slightly above and below the mean frequency of an excited state can still excite the same state without violation of conservation of energy-a mystery explored thoroughly in a previous paper without finding the solution offered here. As usual, a better elucidation of the details of light interaction also leads to new insights-especially about the vacuum field. The Appendix summarizes some previous research relevant to this discussion.
\end{abstract}

\section{Keywords}

Photon Size, Photon Energy, Photon Excitation, Vacuum Field, Red Shift

\section{Introduction}

When we talk about a photon, we specify a frequency/wavelength and direction and position, but its physical size is typically not mentioned. It is the atom or molecule that sets the cross-section for a photon interaction not the photon itself. Given this gap in the physics of photons, we have explored here the question of the size/width of a photon perpendicular to its direction of propagation-defined as the size of a hole that will pass the photon with at least $90 \%$ probability.

While this question is simple and direct, the answer to this question opens a doorway to understanding decades of past research by many groups around the 
world showing annual variations in radioactive decay rates peaking around January each year.

\section{The Logic to Determine the Perpendicular Width of a Photon}

We use the logic shown in Figure 1. We begin with a photon of frequency $f_{1}$. Then the coordinate frame is shifted by velocity $\Delta v$ so that the same photon now has a higher frequency $f_{2}$ given by the relativistic Doppler shift equation as shown in Equation (1).

$$
\lambda=c / f_{2}
$$

Using Equation (1), we can now choose a $\Delta V$ close to the velocity of light $c$ where the photon wavelength $\left(\lambda=c / f_{2}\right)$ is much smaller than the size of a neutron. With such a short wavelength we can then pass the photon through a hole diameter of 1 neutron $=1.6 \times 10^{-15} \mathrm{~m}$ with high transmission and negligible diffraction.

It is especially important to note that with a standard Lorentz transformation the width of the photon perpendicular to its propagation direction will be preserved under all changes in the observer coordinate system. Thus, the width of a photon will be the same in any coordinate system.

If we choose the velocity increase to be close to the velocity of light, then the wavelength of the photon in the shifted coordinate frame can be as small as we like, and in this case, we select a Doppler shift that reduces the photon wavelength to $10^{-20} \mathrm{~m}$.

With such a small photon wavelength, it is no problem to pass the photon through a hole the size of a neutron or less $\left(1.6 \times 10^{-15} \mathrm{~m}\right)$ with lots of room to spare.

This conclusion can be generalized to all coordinate systems since dimensions perpendicular to the velocity change are invariant with the velocity change. This well-known physical principle implies that the width of the photon perpendicular to its direction will be the same size in all coordinate systems for any velocity shift along the photon path i.e., for all frequencies of the photon.

The conclusion of this short discussion is that the perpendicular width of a photon doesn't change with its frequency and is expected to be as small as $10^{-20}$ meters, based on well-proven Lorentz transformations.

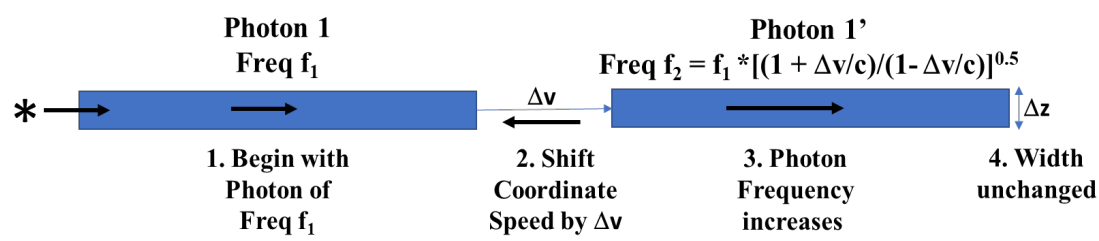

Figure 1. Using a standard Lorentz transform along the direction of an emitted photon, the frequency $f_{1}$ of the emitted photon can be shifted to any value without changing the width of the photon perpendicular to its direction. Thus, the width of a photon is invariant in all coordinate frames and all photon frequencies. 
This extremely small size suggests that a photon has no energy, but interacts with the vacuum field to provide energy for excitations.

Thus, the above discussion with Figure 1 tells us that the size of a photon perpendicular to its velocity is constant for all wavelengths and is comparable to the shortest wavelength a photon can possess, which likely will be one of the prime constants in physics.

\section{Implications}

\subsection{The Width of a Photon Perpendicular to Its Velocity Would Be a Universal Constant of Extremely Small Size, and Thus the Volume and the Energy of a Photon Would Be Close to Zero}

Even more constraining is that the energy excitation of a photon will not come until the end of the photon string actually arrives at the object irradiated. As an example, a decay time of 10 nanoseconds makes the leading part of a photon emission $3 \mathrm{~m}$ long ( $c \times 10$ nanoseconds) while the photon at the tail is around $10^{-20} \mathrm{~m}$ or so wide and likely a similar dimension in length. As a result, the volume available for interaction in the photon itself appears very small in all three dimensions-perhaps less than $\left(10^{-20} \mathrm{~m}\right)^{3}=10^{-60} \mathrm{~m}^{3}$.

\subsection{With Such a Negligible Volume, the Energy in the Photon May Well Be Considered Zero}

Photons interact all over our labs, and we see energy being released as they are absorbed. However, given that the volume of any photon appears to be essentially zero without any matter in it, we explore here the possibility that the photon is a trigger without any significant energy of its own.

We hypothesize that the function of a photon is not to provide energy, but to initiate a process that provides energy.

To be specific in this discussion we imagine a photon that has traveled a long enough distance after emission so that it has negligible E\&M field around it. It is thus modeled as a $10^{-20} \mathrm{~m}$ diameter string (or smaller) with a photon seed at the end containing negligible and possibly zero energy.

However, we know that a photon is able to excite an electronic state as we see in our labs all the time. Assuming no significant energy in the minuscule photon volume, the excitation process from the incident photon must emit negative energy E\&M radiation in order to generate positive energy for the excitation. Since we often model E\&M fields as complex, emitting negative energy seems plausible. We are simply looking at the familiar excitation process in greater detail.

\subsection{The Energy Generation Process}

One clue we have is that the energy effect of a photon, when captured, is Planck's constant h times its frequency $v$. Since Planck's constant $h$ is the energy scale factor for the vacuum field, we propose an interaction of a zero-energy photon with the vacuum field to provide the excitation energy. 
We know from quantum mechanics that an excitation of a fixed state always creates the same excited state no matter what its exact input photon frequency. In particular, the same excitation can be generated by a small band of photon frequencies. We thus need a physical theory that supports a fixed excitation energy from a band of input frequencies of light.

Immediately raising this question: When the same excitation is coming from a band of different frequencies, how do we deal with conservation of energy? [1].

To allow the excitation of an electronic state by a band of frequencies, we need three hypotheses:

1) A photon of frequency $v_{p h}$ within the excitation bandwidth of an excitable state can be captured by that electronic state.

2) Since we have found that a photon has no significant energy, the photon capture must stimulate an $\mathrm{E} \& \mathrm{M}$ radiation process of negative energy to generate the excitation energy of the state.

3) For every photonic excitation, this E\&M radiation process can be activated by photons over a range of frequencies to give exactly the same excited state result.

The energy of a photon (meaning its effect on matter) is usually considered to be $\mathrm{h}^{\star}$ frequency, but when we look more closely, at an excitation, we find the paradox that the same electronic excitation is created by a range of photon frequencies, both lower and higher than the nominal state excitation. Most bothersome, is that this excitation can even be accomplished by photon frequencies where $h v$ is less than the excitation energy. The experimental result is that the energy to excite a state is the same for all exciting photons no matter what their frequency, which means that the excitation energy doesn't come from the photon, which is just a trigger to the excitation process.

How is conservation of energy preserved?

This issue was raised in reference [1], which evaluated several candidate explanations without finding a solution.

Since the probability of receiving a photon that exactly matches the energy of an excitation state change is almost zero, the well-known principle that a band of incident photon frequencies will all cause the same excitation of an electronic state is essential for our universe to be active. However, the photons whose frequencies are closest to the excitation energy will have the highest interaction cross-section.

This discussion concludes that the excitation energy cannot come from the photon, whose function is simply to activate the excitation process.

\subsection{Proposed Model of a Photonic Interaction}

Now return to a photon in the lab interacting with an atom. The physical model we have now derived for a photon excitation in an atom is summarized here.

1) When an incident photon interacts with an atomic state, it will have a frequency close to an available excitation transition but no energy in its negligible 
volume.

2) The photon absorption by the atomic state triggers a negative energy emission from the current atomic state in the same direction as the incident photon at the frequency of the excitation.

3) That negative energy emission generates the positive excitation energy of the electronic state as well as the momentum and angular momentum changes.

4) Some later time, that state decays when it captures a vacuum photon at the proper frequency range, releasing a positive energy E\&M emission that provides the resulting momentum kick.

5) Both the negative and positive energy E\&M emissions diffuse into the background of space, adding to the vacuum field whose total energy is zero.

With this model, energy is fully conserved and these processes will continually increase the vacuum field in the universe.

\subsection{Interim Discussion}

We have presented a simple logic in this paper concluding that all photons have essentially zero volume and thus zero energy. This means that the excitation of a state occurs not from the photon's energy but from the photon's trigger. In our proposed model, the triggered state then emits negative energy E\&M radiation until the electron is excited to the higher state-always creating the same excitation energy no matter what the exact frequency may be of the photon that triggered the excitation process.

This process matches the experimental observations where a small frequency range of incident photons can all excite the same electronic state. This process also resolves the problem of the different frequencies of light all having the same excitation energy since the energy of excitation comes from a negative energy emission of the activated electronic state not from the photon, which is shown above to have no significant energy at all [1].

This process of having a triggered excitation generate its own energy may be universal. As a result, there would be perfect conservation of energy in the universe even when Planck's constant times the frequency of the incident light is too low or too high for the exact energy boost required to lift an electron or nuclear particle into a higher state. And the traditional $h v$ exciting photon energy is almost always too low or too high given the continuous spectra of photons in the universe.

\subsection{Impact on the Cosmos}

This model requires that the E\&M radiation from all sources mostly dissipates into the cosmos, where it has been accumulating since the start of the universe. This radiation would include all the positive energy radiated when an excited state decays as well as the negative energy radiated when a state gets excited in nearly equal proportions. 
The resulting radiation, having accumulated over the lifetime of the universe, would be immensely powerful and zero mean energy-making us consider the vacuum field as its possible result.

If the vacuum field does come from these universal excitations and emission processes, then we would expect to see the level of the vacuum field slowly increasing with time. Since the vacuum field controls the rate of time, we would also see a universal red shift with distance, caused not by an expanding universe but by a universe whose time rate is slowly increasing due to the vacuum field slowly increasing.

We have called this vacuum field radiation by the name "Casimir radiation" since its accumulation sets the value of vacuum field, which Casimir first discovered.

Sources of Vacuum Field Radiation (Casimir Radiation): Stars would be especially strong sources of this vacuum radiation, although every warm body would be a source. Previous tunnel diode experiments [2] [3] detected this vacuum field radiation from the Sun at 13 sigmas and from a nearby source in our galaxy (consistent with Betelgeuse) at 7 sigmas using Esaki tunnel diode current at constant voltage and temperature to measure the changes in the vacuum field. That tunnel current is exponentially sensitive to h, Planck's constant, that traditionally controls the strength of the vacuum field.

See the Appendix for more details about this experiment.

We also detected a gradient in h across the Earth's orbit of $851 \mathrm{ppm}$, peaking in the second half of December (Figure 2).

In this paper, we assume the reverse relationship, that the radiation strength of the vacuum field determines $h$, which can vary spatially with local radiation coming from nearby stars. We also assume that such radiation comes from all warm bodies but dominantly from stars, as does almost all other radiation in the universe.
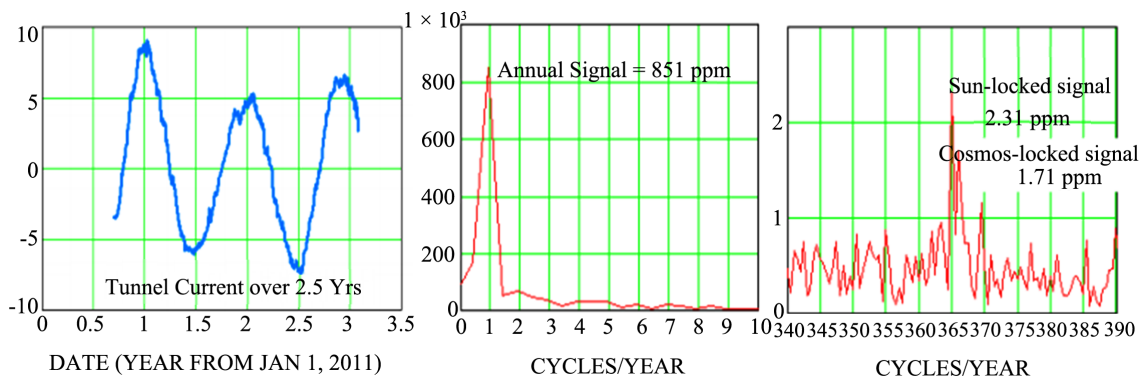

Figure 2. An Esaki tunnel diode experiment, carefully controlled for temperature and isolated from local fields, showed both annual and daily variations in the tunnel current. The annual variations were interpreted as a variation in the Planck parameter $h$ across the Earth orbit. The daily frequency variations had two components associated with the Earth's rotation: One locked to the Sun at $10^{-13}$ false alarm probability and a second locked to a direction in the cosmos at $10^{-7}$ false alarm probability. The first has 365 cycles per 365 days and the second 366 cycles per 365 days over two years, so the data plotted above separated the two signals well. 
The previous tunnel diode experiment measured [1]:

1) The sinusoidally varying tunnel current across the Earth orbit (1 cycle per year).

2) The sinusoidally varying tunnel current aligned to the Sun (365.24 cycles per year).

3) The sinusoidally varying tunnel current from a star in the nearby universe $(365.24+1$ cycles per year).

We found high probability detections from all three effects as shown in the data plotted below.

Note that Casimir radiation from a nearby star is easy to separate from Casimir radiation from our Sun since the signal from the remote star has one extra rotation of the Earth per year, compared to the Sun. More specifically, the Earth's rotation around the Sun subtracts one rotation from inertial space rotation.

Thus, we expect 365 signal oscillations per year for solar radiation and 366 signal oscillations per year for a remote star source. Both were clearly seen at high confidence as shown in Figure 2 [2].

These data show that there are radiative sources for the vacuum field. This current paper provides a physical mechanism for the previously detected vacuum field radiation in the continuous excitations and emissions of electronic states.

In support of this model, we have used data from a 2.5 year tunneling experiment, showing an increase in the tunnel current on the sun-side of the Earth and a larger variation across the Earth's orbit, peaking in December-January. In addition, a third variation was found roughly aligned with Betelgeuse-suggesting that the red giant Betelgeuse may be a substantial source of Casimir radiation.

The model that explains all these variations has the time rate of the universe steadily increasing as the vacuum field increases from the continuing photonic interactions of stars and other matter. Such a universe could show the same red shift with distance that we see today but due to the increasing vacuum field with time and not due to an expanding universe.

The left side of Figure 2 shows the 2.5 year history of the tunnel current in the Esaki tunnel diodes-chosen for their extreme stability. Assuming there might be a transient in the start up of the experiment, the last two years of the data were used in the analysis.

There were three different frequencies of signal detected in this experiment [2].

1) One year cycle: The largest detected signal showed a gradient in h of 851 ppm across the Earth's orbit.

Peaking in the second half of December.

2) 24-hour Cycle: $2.31 \mathrm{ppm}$ (Probability of Random chance $10^{-13}$ ) locked to the Sun at 365 cycles per year. This would correspond to Casimir radiation from the Sun (9.5 sigma). 
3) There was also a daily signal of $1.71 \mathrm{ppm}$ (7 sigma) (Probability of Random chance $10^{-7}$ ) locked to a direction in the universe consistent with Betelgeuse-a gigantic red star brightly seen every night. It is clearly separable from the 24 hours cycle since it has one extra cycle per year, two extra cycles in the two years of data.

\subsection{Gradient across the Earth Orbit}

The strongest signal detection at $851 \mathrm{ppm}$ peak-to-valley was a signal with one year period, which would correspond to a gradient in the vacuum field (i.e., a gradient in $h$ ) across the Earth's orbit, peaked in the second half of December. Such a gradient would come from a remote and powerful source.

\subsection{Sun Source}

A frequency analysis of this data over two years also detected a daily frequency (365 cycles per year) locked to our Sun at $2.31 \mathrm{ppm}$ peaking around local noon. This would correspond to Casimir radiation (vacuum field) coming from the Sun.

Probability of random event $=1.05 \times 10^{-13}$.

\subsection{Star Source}

In addition, a weaker source for the vacuum field of $1.71 \mathrm{ppm}$ was detected with a frequency of 366 cycles per year consistent with a remote star source, generally aligned with the giant red star Betelgeuse. It is weaker in our solar system because Betelgeuse is very far away compared to our Sun.

Probability of random event $=1.0 \times 10^{-7}$.

\section{Conclusions}

Impact on the Cosmic Red Shift: If the vacuum field is radiated by all stars, the rate of time is speeding up everywhere as the universe ages. The effect of a steadily increasing rate of time would be that stars further away appear redder than stars close to us, and the red shift will be roughly linear in distance-matching the observed red shift. The physics for the well-known cosmic red shift in this new model is simply a steady increase in the vacuum field with time-radiated continuously by every star and warm object in the universe. Even more, this radiated vacuum field seems to be required to explain a gradient in tunnel current across our Earth orbit and an enhanced tunnel signal on the sun side of the Earth as experimentally observed.

Impact on radioactive decay: This paper shows a 2.5 year history of annual variations in tunnel current, also suggesting that radioactive decay rates would be affected-both especially sensitive to the vacuum field. While the tunnel current experiments were done by the author, the radioactive decay experiments were done by different groups around the world. Siegert [4] published 15 years of data showing radioactive decay of ${ }^{226} \mathrm{Ra}$ peaking around Jan 30 each year. Al- 
burger et al. [5] found ${ }^{32} \mathrm{Si}$ decay peaked around Feb 8 in 4 years of data. Parkamov [6] found annual variations in the radioactive decay of ${ }^{60} \mathrm{Co}$ and also a mixture of ${ }^{90} \mathrm{Sr}^{90} \mathrm{Y}$, peaking in January to February of each year. The number of time varying decay reports caught DARPA's interest, so Ref [7] was funded by DARPA to assemble the many experimental results related to time varying radioactive decay in the hope that someone could integrate them into a single theory.

The results of this paper all applied to tunnel current and a low frequency vacuum field of $h v$ about $0.3 \mathrm{eV}$. The annual and daily variations in tunnel current were well suited to explore some new physics of the vacuum field. The outcome was a candidate explanation for the cosmic red shift that requires no Big Bang-just a lot of hot stars.

\section{Conflicts of Interest}

The author declares no conflicts of interest regarding the publication of this paper.

\section{References}

[1] Hutchin, R.A. (2015) The Uncertain Energy of an Excited State. Optics and Photonics Journal, 5, 201-204. https://doi.org/10.4236/opj.2015.56019

[2] Hutchin, R.A. (2019) Detection of Casimir Radiation from Our Sun. Optics and Photonics Journal, 9, 141-154. https://doi.org/10.4236/opj.2019.99013

[3] Hutchin, R.A. (2016) Experimental Evidence for Variability in Planck's Constant. Optics and Photonics Journal, 6, 124-137. https://doi.org/10.4236/opj.2016.66015

[4] Siegert, H., Schrader, H. and Schötzig, U. (1998) Half-Life Measurements of Europium Radionuclides and the Long-Term Stability of Detectors. Applied Radiation and Isotopes, 49, 1397-1401. https://doi.org/10.1016/S0969-8043(97)10082-3

[5] Alburger, D.E., Harbottle, G. and Norton, E.F. (1986) Half-Life of ${ }^{32}$ Si. Earth and Planetary Science Letters, 78, 168-176. https://doi.org/10.1016/0012-821X(86)90058-0

[6] Parkhomov, A.G. (2010) Researches of Alpha and Beta Radioactivity at Long-Term Observations. http://arxiv.org/abs/1004.1761

[7] McDuffie, M.H., Graham, P., Eppele, J.L., Gruenwald, J.T., Javorsek II, D., Krause, D.E. and Fischbach, E. (2020) Anomalies in Radioactive Decay Rates: A Bibliography of Measurements and Theories. 


\section{Appendix}

Two Charts from OSA Presentation [2]: Casimir Radiation Detected from the Sun at 13 Sigmas by Richard A. Hutchin.

Figure A1 shows the fully assembled system in its shielded cage. The five parallel Schottky diodes shown in Figure A2 are in the shielded cage on the right side, keeping them well isolated from the power supplies and the data acquisition electronics.

The 5 Schottky tunnel diodes (Figure A2) were attached to a thick, temperature controlled, copper plate using thermally conducting glue, and the temperature of the plate (as well as many other temperatures in the system) were monitored every 10 seconds along with all the currents and voltages. We had previously calibrated tunnel diode current versus temperature and removed the small effect in the processing as the internal temperature shifted slightly. That temperature was set to over $40^{\circ} \mathrm{C}$ so that it could be maintained closed loop by adding heat to the thick copper plate that supported all the tunnel diodes.

A constant current (separately measured every 10 seconds) was applied to the 5 Schottky tunnel diodes in parallel, and the resulting voltage was measured and recorded also every 10 seconds for 2.5 years.

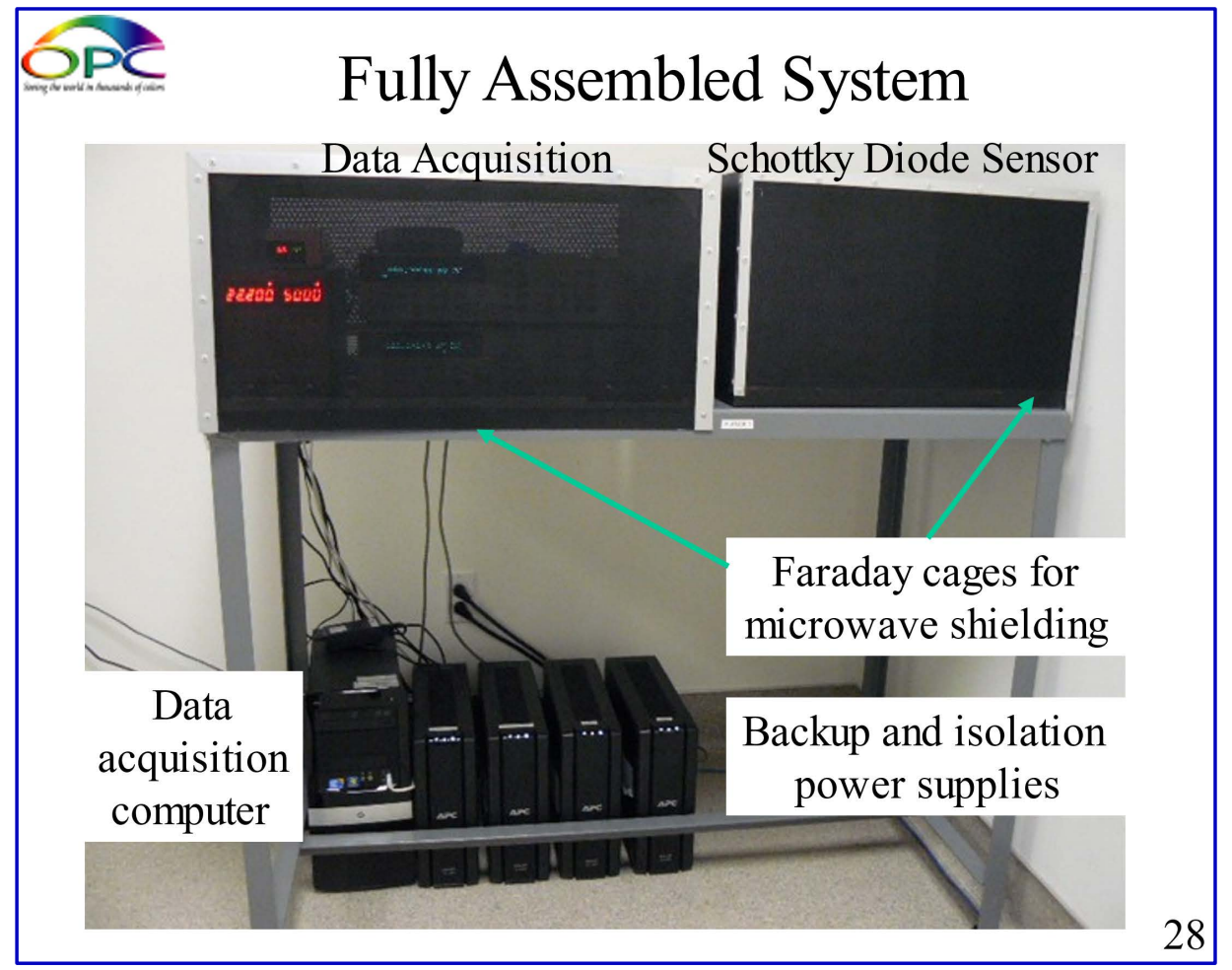

Figure A1. This picture shows the Esaki tunnel diode experiment that operated in our lab for 2.5 years measuring the vacuum field intensity versus time of day and time of year. The output of this experiment is shown above in Figure 2. All the power regulation and data acquisition equipment were installed in the left Faraday cage while the sensor head with its 5 parallel Schottky diodes was in its own shielded cage on the right. All temperatures, voltages, currents and signals were archived every 10 seconds for 2.5 years. 


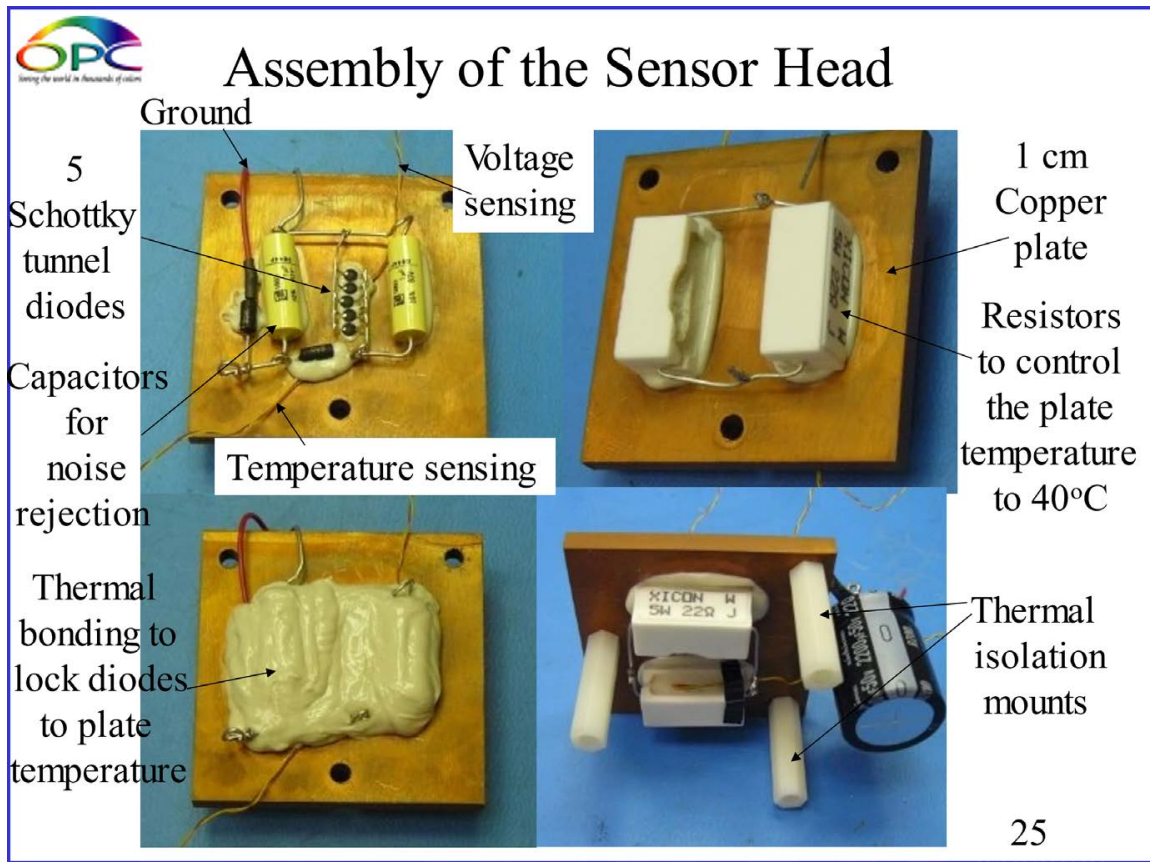

Figure A2. All critical sensing components were in their own isolated and shielded box. The 5 Schottky tunnel diodes (known for their stability) were used to measure the shift in Planck's constant $h$ by their current fluctuations. Tunnel diodes are exponentially sensitive to $h$. 